\title{
Aplicação da técnica de sondagem em freqüência na caracterização de canais de UWB exteriores
}

\author{
Christiano F. Souza, Julio Cesar R. Dal Bello
}

\begin{abstract}
Resumo - Este artigo apresenta a aplicação da técnica de sondagem em freqüência na caracterização de canais UWB. Um canal de $750 \mathrm{MHz}$ de largura de banda foi investigado e os perfis de retardos de potência foram obtidos a partir dos dados medidos com o auxílio da técnica CLEAN e da janela de Blackman-Harris de 3 termos. A partir deles, os parâmetros de dispersão temporal do canal foram calculados. $O$ relacionamento entre o espalhamento de retardos e a banda de coerência também foi estudado.
\end{abstract}

Palavras-chave - Caracterização de canal, sondagem em freqüência, UWB.

Abstract - This article presents the sounding frequency technique applied to the characterization of UWB outdoor channels. A $750 \mathrm{MHz}$ bandwidth channel was surveyed as an example. The power delay profiles were carried out from the measure data with the help of CLEAN technique and the 3 terms Blackman-Harris window. From the measurements, the channel temporal dispersive parameters were obtained. The relationship between the delay spread and the coherence bandwidth were also studied. UWB.

Keywords - Channel characterization, frequency sounding,

\section{INTRODUÇÃO}

Ainda são poucas as referências de campanhas de medidas de sinais UWB em ambientes externos. A primeira delas foi realizada por Win et al [1], em 1997, onde foi sondado no tempo um canal de $1,3 \mathrm{GHz}$ de largura de banda com pulsos transmitidos a cada $500 \mathrm{ns,} \mathrm{em} \mathrm{uma} \mathrm{área} \mathrm{rural.}$

Os trabalhos de Di Francesco [2] e de Di Renzo [3] também tratam da sondagem no tempo de canais UWB, em ambientes externos, sendo o primeiro em um ambiente suburbano e o segundo em dois ambientes: um de floresta e outro em terreno montanhoso. No trabalho de Kim [4], o canal UWB é sondado no domínio da frequiência, em dois ambientes externos, em LOS (Line of Sight) e em NLOS (Non Line of Sight).

Este artigo tem por objetivo apresentar o uso da técnica de sondagem em frequiência na caracterização de canais de Banda Ultra Larga (UWB). Para isso, será apresentado o resultado de uma campanha de medidas realizada no campus da Praia Vermelha da Universidade Federal Fluminense (UFF), em um ambiente exterior. Os perfis de retardos de potência foram obtidos a partir dos dados adquiridos; os parâmetros de dispersão temporal do canal (espalhamento de retardos e banda de coerência) foram calculados a partir destes perfis e seus relacionamentos foram analisados através do limiar de Fleury [5] e do ajuste de Gans [6]. O resultado completo desta campanha de medidas está disponível em [7].

Este artigo está organizado como se segue: Na Seção 2 é apresentado o Sistema de Banda Ultra Larga (UWB). Nas Seções 3 e 4 são descritos o ambiente sondado e o setup de medidas, respectivamente. A Seção 5 apresenta o funcionamento da técnica de sondagem em freqüência, e, a Seção 6, apresenta os detalhes do processamento de sinais realizado. Na Seção 7, os resultados das medidas e as análises correspondentes são apresentados. Finalmente, a Seção 8 conclui o artigo.

\section{OS SISTEMAS DE BANDA ULTRA LARGA (UWB)}

Um sistema UWB é definido como sendo qualquer sistema de transmissão sem fio que ocupe uma largura de banda maior do que $500 \mathrm{MHz}$ ou uma largura de banda fracional $\mathrm{B} / \mathrm{f}_{\mathrm{c}} \geq 0,20$. Nesta relação, $\mathrm{B}$ é a largura de banda espectral dada por $B=f_{f}-f_{i}$, em que $f_{f}$ e $f_{i}$ são as freqüências final e inicial, respectivamente, medidas nos pontos de $10 \mathrm{~dB}$ do espectro, e $\mathrm{f}_{\mathrm{c}}$ é a freqüência central na banda [8].

Os sistemas UWB apresentam uma série de importantes vantagens, como destaca Molisch [9]: permitem a localização mais precisa de espalhadores e possuem maior robustez a desvanecimentos por multipercursos, devido à pequena resolução temporal; permitem múltiplo acesso devido às amplas bandas de transmissão; possibilitam taxas de transmissão extremamente altas; permitem comunicações sigilosas devido às baixas potências transmitidas.

\section{AMBIENTE EXTERNO MEDIDO}

O ambiente no qual as medidas foram realizadas está ilustrado na figura 1 .

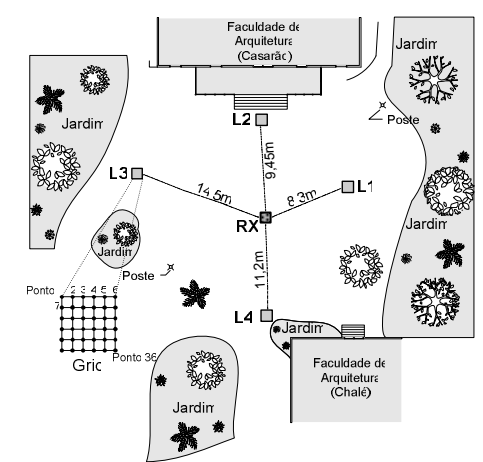

Fig. 1. Ambiente exterior medido. 
A área investigada é aberta, cercada de arbustos e de algumas árvores grandes, e o piso é feito de paralelepípedo.

As medidas foram realizadas em um domingo à tarde para evitar a contaminação das mesmas, com desvio Doppler, pelo deslocamento das pessoas. Cuidados especiais foram adotados para que os pesquisadores não obstruíssem a linha de visada (LOS) e nem interferissem na estacionariedade do ambiente. Assim, neste trabalho, considerou-se o canal como sendo WSSUS (Wide Sense Stationary with Uncorrelated Scatters).

\section{SETUP DE MEDIDAS}

O setup de medidas utilizado foi composto pelos seguintes equipamentos: um analisador de rede vetorial, um amplificador de baixo ruído, duas antenas discônicas, um computador, uma fonte de tensão de 12 VCC, cabos coaxiais e conectores. A figura 2 mostra o setup utilizado nas medidas.

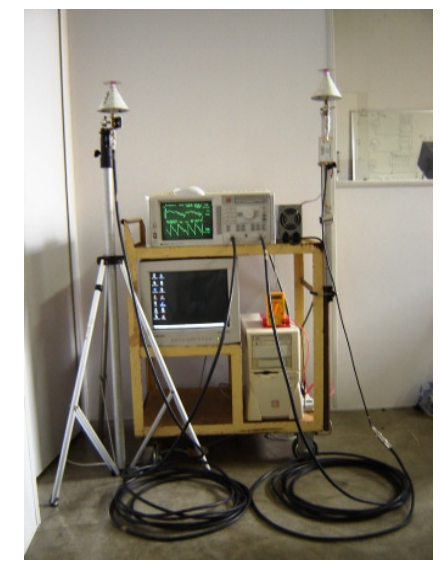

Fig. 2. Setup utilizado na sondagem em freqüência.

O analisador gera um sinal de RF que é aplicado ao dispositivo sob análise (DUT - Device Under Test), que neste caso foi o próprio canal rádio-móvel, com o uso de duas antenas, uma para transmissão e outra para recepção, conectadas aos terminais de saída e de entrada do analisador, respectivamente.

Para se sondar o canal, várias senóides são geradas sucessivamente pelo analisador, em cada uma das frequiências discretas que compõem a banda de interesse, "varrendo-a". É por este motivo que esta técnica de sondagem é também conhecida como Técnica de Varredura em Frequiência. Como o sinal gerado é conhecido, a priori, informações de amplitude, fase e retardo de grupo podem ser obtidas indiretamente a partir do parâmetro $\mathrm{S}$, conforme a equação abaixo:

$$
\mathrm{S}=10 \cdot \log _{10} \frac{\mathrm{P}_{\text {in }}}{\mathrm{P}_{\text {out }}}
$$

Após a propagação pelo canal, o sinal é adquirido por uma antena receptora conectada a um LNA (Low Noise Amplifier), que está ligado na porta de entrada (porta de recepção) do analisador, para ser processado e analisado. Desta forma, as amplitudes e fases do sinal na recepção podem ser aquisitadas. Estes valores de amplitudes adquiridos representam uma relação entre as potências de entrada e de saída, o que caracteriza a função de transferência T do canal, conforme Parsons [10].

Um computador é utilizado para armazenar os dados medidos e controlar as varreduras. O LNA serve para dar ganho ao sinal, na recepção. A fonte de 12 VCC alimenta o LNA. Os cabos coaxiais e conectores são utilizados na interconexão dos equipamentos de RF.

O computador é conectado ao analisador por meio de um cabo HPIB (Hewlett Packard Interface Bus) e uma placa de aquisição específica, também da HP, cujo protocolo de comunicação foi criado via um programa em Matlab. Este programa, portanto, é o responsável pelo controle das varreduras e pela aquisição e armazenamento dos dados.

As antenas banda-larga utilizadas são do tipo discônicas, onidirecionais no plano horizontal. A distância entre o plano de terra e a carga de topo da antena foi mantida em $1 \mathrm{~cm}$, para um dielétrico de $\varepsilon_{\mathrm{r}} \approx 1$, pois a perda de retorno foi a mais constante possível, dentro da banda investigada. Nesta banda, o ganho das antenas é de 2,14 dBi, e a perda de retorno, em média, abaixo de $-15 \mathrm{~dB}$. As antenas foram mantidas a 1,5 metro do solo, em todas as medidas.

O LNA utilizado, fabricado pela MINI-Circuits, modelo ZLR-2150, tem faixa de amplificação de $950 \mathrm{MHz}$ a 2150 $\mathrm{MHz}$, ganho de $25 \mathrm{~dB}$ na faixa de $950 \mathrm{MHz}$ a $1800 \mathrm{MHz}$, e funciona com uma tensão de alimentação contínua de 12 VCC. O LNA é um elemento importante para se ter um sinal mais forte na recepção, sobretudo devido à atenuação de espaço livre e perdas em conectores e cabos coaxiais.

Os cabos coaxiais utilizados são do tipo RG-213 e RG$58 \mathrm{U}$ interligados ao restante do setup por meio de conectores tipo $\mathrm{N}$ e SMA.

\section{TÉCNICA DE SONDAGEM EM FREQÜÊNCIA}

A técnica de sondagem no domínio da freqüência empregou um Analisador de Rede Vetorial HP8714ET como transceptor para a aquisição das amplitudes e fases de 1601 pontos discretos, tomados dentro de uma banda de frequiências, excitando-se uma portadora senoidal em tais pontos. A banda escolhida foi de $960 \mathrm{MHz}$ a $1710 \mathrm{MHz}$, resultando em uma banda de $750 \mathrm{MHz}$. A medição desta banda, em vários pontos do ambiente, permite a determinação da função $\mathrm{T}(\mathrm{f}, \mathrm{t})$ do canal, da forma indicada na equação 2 :

$$
[\mathrm{T}]_{\mathrm{f} \times \mathrm{t}}=[\mathrm{A}]_{\mathrm{f} \times \mathrm{t}} \times\left[\cos \left([\mathrm{F}]_{\mathrm{f} \times \mathrm{t}} \times \frac{\pi}{180}\right)+\mathrm{j} \cdot \operatorname{sen}\left([\mathrm{F}]_{\mathrm{f} \times \mathrm{t}} \times \frac{\pi}{180}\right)\right]
$$

em que $[\mathrm{A}]_{\mathrm{fxt}}$ e $[\mathrm{F}]_{\mathrm{fxt}}$ representam as amplitudes e fases para cada ponto de freqüência discreta dentro da banda utilizada na sondagem. Neste trabalho, todavia, o tempo " $t$ " foi mantido fixo, correspondendo a uma "varredura". Assim, a função $T(f, t)$ obtida é composta apenas de amostras em freqüências discretas dentro da banda sondada.

Como o Analisador de Rede Vetorial está sempre comparando um sinal transmitido, conhecido à priori, com o sinal recebido do canal, os efeitos dos dispositivos a ele 
ligados não devem influenciar na medida final. Isto é conseguido através do processo de calibração do analisador, após o qual ele incorpora os efeitos destes dispositivos, compensando suas perdas, sendo esta uma funcionalidade já disponível internamente no mesmo.

A calibração é normalmente feita através da ligação direta de todos os equipamentos que compõem o setup ao Analisador de Rede Vetorial. Contudo, não é possível tal ligação em um sistema conectado a duas antenas. A calibração, então, foi feita conforme sugerida por Zaghloul [11], com a ligação direta dos cabos e conectores utilizados, entre as portas de entrada e saída de RF do analisador, sem as antenas. A ausência das antenas no processo de calibração introduziu um retardo, de aproximadamente $2 \mathrm{~ns}$, devido à propagação do sinal nas antenas de transmissão e de recepção.

Com a calibração realizada, há uma mudança nos pontos de referência de tempo, da porta do analisador para os pontos de calibração localizados no final dos cabos (conectores) que são ligados às antenas. Assim sendo, as informações de amplitude e fase recebidas passam a ser exclusivamente do canal rádio-móvel.

Em todas as medidas realizadas, a potência de saída do analisador foi mantida em $10 \mathrm{dBm}$, que se mostrou suficiente para difundir o sinal pelo ambiente e ser recebido com a intensidade necessária para o pós-processamento.

Estando o setup devidamente calibrado e pronto para a medição, a varredura de uma banda $\mathrm{B}$ com $\mathrm{N}$ pontos igualmente espaçados pôde ser iniciada, sendo o espaçamento em freqüência dado por $\Delta \mathrm{f}=\mathrm{B} /(\mathrm{N}-1)$. Nesta técnica, as medidas são realizadas no domínio da freqüência e, assim, obtém-se a função T(f,t), como definida pela equação 2 .

As respostas no domínio dos retardos, dadas pela função $\mathrm{h}(\mathrm{t}, \tau)$, são obtidas, indiretamente, através de uma Transformada Rápida de Fourier Inversa, aplicada ao vetor que define $[\mathrm{T}]_{\mathrm{fxt}}$, conforme Parsons [10].

$\mathrm{O}$ retardo máximo $\tau_{\mathrm{MAX}} \mathrm{e}$ a resolução temporal $\Delta \tau$ conseguidos são resultantes da configuração do analisador, adotada durante as medidas. $O$ retardo máximo $\tau_{\mathrm{MAX}}$ representa o maior valor de retardo que se consegue medir, e está associado ao sinal resultante do somatório dos multipercursos que percorrem a maior distância entre os pontos de transmissão e recepção. A resolução temporal $\Delta \tau$ indica a diferença mínima no tempo de chegada entre dois raios, para que estes possam ser distinguidos pelo analisador. Em outros termos, $\Delta \tau$ representa a menor diferença entre as distâncias percorridas por dois raios, para a qual o analisador ainda consegue diferenciá-los.

Pelas propriedades da transformada de Fourier [12], temos as seguintes correspondências entre os domínios da frequiência e dos retardos.

$$
\begin{aligned}
& \Delta \tau=\frac{1}{\mathrm{~B}} \\
& \tau_{\mathrm{MAX}}=\frac{1}{\Delta \mathrm{f}}=\frac{\mathrm{N}-1}{\mathrm{~B}}
\end{aligned}
$$

Os valores configurados no analisador e os parâmetros resultantes adotados na campanha de medidas realizada (sondagem) encontram-se resumidos na tabela 1.

\begin{tabular}{|l|c|c|}
\hline \multicolumn{1}{|c|}{ Parâmetros } & Valor & Unidade \\
\hline Faixa de freqüências & $960-1710$ & $\mathrm{MHz}$ \\
\hline Largura de banda & 750 & $\mathrm{MHz}$ \\
\hline $\begin{array}{l}\text { Espaçamento em freqüência } \\
\text { entre amostras }(\Delta \mathrm{f})\end{array}$ & 0,46875 & $\mathrm{MHz}$ \\
\hline Número de amostras varridas & 1601 & - \\
\hline Resolução temporal $(\Delta \tau)$ & 1,33 & $\mathrm{~ns}$ \\
\hline Retardo máximo $\left(\tau_{\mathrm{MAX}}\right)$ & 2133 & $\mathrm{~ns}$ \\
\hline Tempo de varredura & 696 & $\mathrm{~ms}$ \\
\hline Potência de transmissão & +10 & $\mathrm{dBm}$ \\
\hline Ganho do LNA & 25 & $\mathrm{~dB}$ \\
\hline Ganho das antenas & 2,14 & $\mathrm{dBi}$ \\
\hline \multicolumn{2}{|c|}{ Tabela 1. Configuração do analisador de rede e parâmetros da } \\
\multicolumn{2}{|l}{ sondagem } \\
\hline
\end{tabular}

A resolução $\Delta \tau=1,33 \mathrm{~ns}$ significa que componentes de multipercurso separadas de $40 \mathrm{~cm}$ podem ser distinguidas pela sondagem. O tempo máximo de observação da resposta ao impulso, ou seja, o retardo máximo observado, $\tau_{\mathrm{MAX}}=$ 2133 ns permite que percursos com até $640 \mathrm{~m}$ possam ser detectados.

O tempo de varredura do analisador, configurado através da função auto sweep, foi mantido em $696 \mathrm{~ms}$, representando o menor tempo de varredura que o equipamento consegue fornecer para a faixa de frequiências varrida e número de pontos tomados.

O tempo de varredura (696 ms) e o espaçamento em frequiência entre as amostras $(0,47 \mathrm{MHz})$, resultantes da escolha da faixa de frequiências $(750 \mathrm{MHz})$ e do número de pontos para amostragem (1601), mostraram-se adequados para o não comprometimento das estatísticas de banda de coerência e de estacionariedade do canal, durante as medidas. Em outras palavras, nem o espaçamento de frequiências foi grande, para não comprometer o cálculo da banda de coerência, nem o número de pontos (amostras) adotado acarretou um tempo de varredura que violasse as premissas de estacionariedade do canal [7].

A técnica de sondagem do canal em freqüência é, contudo, criticada por não apresentar uma medida de tempo real, conforme observa Rappaport [13]. O argumento é que a completa varredura da banda de interesse exigiria um determinado tempo, durante o qual a resposta em freqüência do canal, sabido ser variante no tempo, poderia mudar rapidamente, resultando em uma medida errônea da resposta ao impulso. No entanto, esta técnica está de acordo com a teoria clássica e vem sendo utilizada por diversos autores, [24], [14]. Para pequenos intervalos de tempo ou para pequenos deslocamentos do móvel, o canal pode ser considerado invariante no tempo, onde as estatísticas de desvanecimento não mudam dentro de uma pequena janela de tempo de observação.

\section{PÓS-PROCESSAMENTO}

O perfil de retardos de potência é obtido a partir de uma transformada inversa rápida de Fourier (IFFT) aplicada ao 
vetor $[\mathrm{T}]_{\mathrm{fxt}}$, definido na equação 2 . Este processo causa um truncamento no domínio dos retardos pois as informações de amplitude e fase do sinal, emitidas pelo analisador, são tomadas dentro de uma faixa de freqüências limitada.

$\mathrm{O}$ primeiro cuidado que se deve ter com a aplicação da IFFT é que esta considera que o sinal começa na freqüência zero. Neste trabalho, o sinal $[\mathrm{T}]_{\mathrm{fxt}}$ encontra-se deslocado e limitado entre as freqüências $960 \mathrm{MHz}$ e $1710 \mathrm{MHz}$. Sabe-se que o deslocamento de uma função na freqüência faz aparecer uma fase na função temporal, conforme Lathi [15].

$$
\mathrm{f}(\mathrm{t}) \mathrm{e}^{-\mathrm{j} \varpi_{0} \mathrm{t}} \leftrightarrow \mathrm{F}\left(\bar{\varpi}-\bar{\varpi}_{0}\right)
$$

A teoria de processamento de sinais ensina que a transformada direta de um sinal real (FFT) tem um gráfico espectral de amplitude par e um gráfico espectral de fase ímpar. A IFFT de $[\mathrm{T}]_{\mathrm{fxt}}$ fornece $\mathrm{h}(\mathrm{t}, \tau)$. Portanto, caso $\mathrm{h}(\mathrm{t}, \tau)$ fosse real, dever-se-ia completar a função $[\mathrm{T}]_{\mathrm{fxt}}$ com zeros até a freqüência de $960 \mathrm{MHz}$ e fazer um espelhamento com o conjugado deste resultado. Só então, poder-se-ia aplicar a IFFT. Esta situação está exemplificada na figura 3.

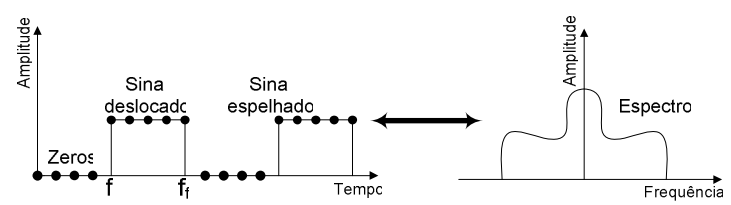

Fig. 3. Procedimentos para se aplicar a IFFT.

No entanto, a aplicação da IFFT sobre a função $[\mathrm{T}]_{\mathrm{fxt}}$ medida pode ser feita diretamente, sabendo-se que sua resposta $\mathrm{h}(\mathrm{t}, \tau)$ aparecerá com uma fase correspondente à freqüência deslocada do início da banda, que neste caso é 960 MHz. Todavia, a obtenção dos parâmetros de dispersão temporal do canal não requer a informação de fase da resposta ao impulso. Desta maneira, trabalha-se unicamente com o módulo da envoltória complexa da resposta ao impulso, resultante da aplicação da IFFT sobre a função $[\mathrm{T}]_{\mathrm{fxt}}$. Vale destacar que a resposta ao impulso do canal $\mathrm{h}(\mathrm{t}, \tau)$ é complexa, resultante dos vários multipercursos que chegam com fases aleatórias no receptor.

A função $[\mathrm{T}]_{\mathrm{fxt}}$ medida neste trabalho, no entanto, já representa uma função discretizada no domínio da frequiência, cujo modelo matemático equivale à multiplicação do espectro de um sinal por uma função janela retangular, representando um filtro passa-banda. Assim, quando se aplica a IFFT na função $[\mathrm{T}]_{\mathrm{fxt}}$, ocorre o aparecimento de espúrios de sinal no domínio dos retardos: obtém-se a amplitude da amostra no retardo $\tau$ e amplitudes laterais localizadas em outros valores de retardo. Para uma resolução temporal de $\tau$, os espúrios de duas amostras adjacentes na freqüência separadas por $\Delta \mathrm{f}$ (pontos adjacentes na função $[\mathrm{T}]_{\mathrm{fxt}}$ ) poderão até se somar, resultando em uma determinada amplitude que dê a falsa impressão de se tratar de um multipercurso, mascarando a informação original que se quer obter.

Com o objetivo de se minimizar o efeito destas componentes indesejáveis, o sinal complexo medido $[\mathrm{T}]_{\mathrm{fxt}}$ é multiplicado por uma função janela antes de se realizar a
IFFT para se obter o perfil de retardos de potência $P_{h}(\tau)$. Esta função janela, como destaca Harris [16], atua como um filtro, reduzindo os lóbulos laterais. Contudo, a multiplicação pela função janela a cada ponto discreto de informação do sinal complexo medido (função $[\mathrm{T}]_{\mathrm{fxt}}$ ), apesar de reduzir consideravelmente os lóbulos laterais, causa um aumento no valor da resolução no domínio dos retardos. Este aumento de resolução faz com que alguns pontos, adjacentes ao sinal medido, a depender de suas amplitudes, não sejam diferenciados. A consequiência disto é a perda de alguns multipercursos do perfil de retardos de potência.

Um estudo inicial mostrou que a janela retangular, de melhor resolução, é a que permite que mais multipercursos sejam considerados. Já as outras janelas (Blackman-Harris de 3 e 4 termos e Hanning) permitiam a captura de uma quantidade de multipercursos aproximadamente igual. Aplicar uma janela retangular equivale a multiplicar o sinal a ser "janelado" por 1, o que não é tão eficiente para a retirada dos efeitos de borda de um sinal truncado.

Para as medidas realizadas, a janela de Blackman-Harris de 3 termos foi a que apresentou a maior redução de lóbulo lateral para uma menor perda de resolução entre multipercursos, sendo por isso, utilizada.

$\mathrm{O}$ aumento de resolução inerente à janela de BlackmanHarris de 3 termos não representou uma perda tão significativa para a análise, uma vez que para ambientes mais amplos, os espalhadores estão mais dispersos e distantes uns dos outros, permitindo se ter uma maior resolução temporal.

Apesar do cuidadoso procedimento de calibração do setup já explicado, os perfis de retardos de potência medidos ainda apresentavam algum ruído intrínseco aos equipamentos de medidas, mascarando as informações que são realmente válidas. Assim, para calcular os parâmetros de dispersão temporal do canal, é necessário, antes, obter um perfil de retardos de potência limpo, onde fiquem evidenciados, apenas, os percursos válidos, suas amplitudes e retardos. No passado, quando estas técnicas de limpeza não eram estudadas e implementadas, os valores dos parâmetros do canal acabavam sendo superestimados.

Neste trabalho, é assumido que o canal é invariante no tempo durante o período de observação, que corresponde ao tempo de varredura (696 ms). Assim, a resposta ao impulso do canal é da forma [17]:

$$
h(t)=\sum_{k=0}^{N-1} a_{k} \delta\left(t-t_{k}\right) e^{j \theta_{k}}
$$

A saída do canal, devido a um sinal transmitido $\mathrm{s}(\mathrm{t})$ é, então:

$$
y(t)=n(t)+\int_{-\infty}^{+\infty} s(t) h(t-\tau) d \tau
$$

Para extrair a resposta ao impulso do canal, a partir da forma de onda recebida $\mathrm{y}(\mathrm{t})$, é preciso que os multipercursos válidos sejam detectados, o que só será possível pela aplicação de uma técnica de limpeza de perfil. 
A resposta ao impulso do canal é dada por $h(t, \tau)$ e o perfil de retardos de potência, por $|h(\tau)|^{2}$. O resultado imediato obtido pela aplicação da IFFT à função $[\mathrm{T}]_{\mathrm{fxt}}$ representa a envoltória complexa do sinal na recepção, cujo módulo foi submetido à técnica de limpeza CLEAN [17].

$\mathrm{O}$ algoritmo CLEAN é um algoritmo de cancelamento seqüencial de interferências no canal e de erros inerentes aos equipamentos do setup, que se apresentam como um ruído dentro do perfil medido e obtido via IFFT da função $[\mathrm{T}]_{\mathrm{fxt}}$. Este algoritmo parte da premissa básica de que o sinal medido é uma soma de pulsos, com formas conhecidas, sendo apresentado inicialmente em [14], em um contexto de estudo do canal UWB. Atualmente, esta técnica vem sendo bastante utilizada por diversos autores [2-3], [19] nos estudos sobre medições de canais UWB, por sua capacidade, já verificada, em gerar respostas impulsivas confiáveis, do canal.

Resumidamente, o algoritmo encontra o sinal mais forte através da correlação do sinal recebido com uma forma de onda padrão de referência, identificando o maior pico nesta correlação. A amostra que gerou este pico de correlação é subtraída do sinal total, e o pulso é novamente correlacionado com o sinal "limpo", resultante desta subtração. Este procedimento é repetido, iterativamente, até que o nível de sinal no perfil que está sendo limpo fique abaixo de um determinado limiar [20].

A medida para estabelecimento da referência deve ser feita em visibilidade, em um ambiente externo, de preferência sem espalhadores, onde a resposta ao impulso possa conter apenas os efeitos do aparelho transceptor, praticamente sem a contribuição de sinais refletidos. Neste trabalho, esta medida foi realizada no campo de futebol do campus do Gragoatá da Universidade Federal Fluminense, um local bastante amplo, praticamente sem espalhadores. As antenas de transmissão e recepção foram colocadas a um metro de distância uma da outra.

\section{RESULTADOS DE MEDIDAS EM AMBIENTE EXTERNO}

O limiar de ruído foi escolhido, criteriosamente, para cada local medido, em função das distâncias TX-RX e dos espalhadores existentes nas vizinhanças do ponto medido. Alguns autores, no entanto, adotam o procedimento de estabelecer um valor fixo acima do patamar de ruído $(7,5 \mathrm{~dB}$ [21] e $10 \mathrm{~dB}$ [4]) ou abaixo do valor de pico no perfil de retardos de potência $(10 \mathrm{~dB}$ [3] e $25 \mathrm{~dB}$ [22]). Com a definição criteriosa do limiar de ruído para cada local medido conseguiu-se não subestimar nem superestimar os valores dos parâmetros de dispersão temporal do canal.

Os perfis de retardos de potência tiveram um comportamento similar nos quatro locais medidos. A figura 4 ilustra um perfil de retardos de potências para o local L3 que pode ser visualizado na figura 1 .

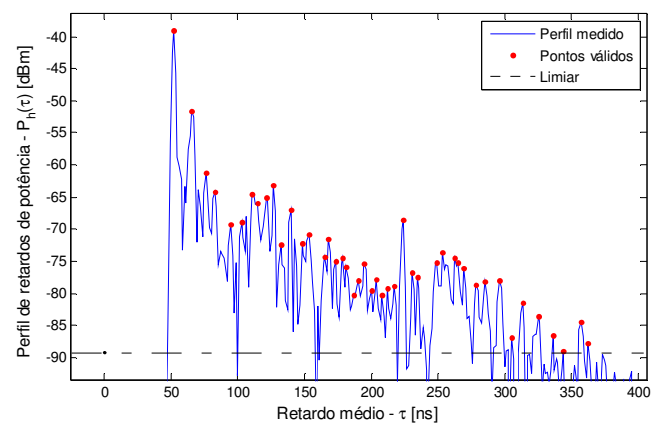

Fig. 4. Perfil de retardos de potência no local L3.

O relacionamento entre a banda de coerência, para os níveis de correlação de 0,7 e 0,9 , e o espalhamento de retardos foi estudado através do limiar de Fleury [5] e da relação de Gans [6], que propôs uma relação da forma $\mathrm{BC}=1 /(\alpha \sigma)$, sendo $\alpha$ uma constante numérica, que permite verificar o quanto a banda de coerência é inversamente proporcional ao espalhamento de retardos $\sigma$. Fleury propôs um limiar inferior na relação entre banda de coerência e espalhamento de retardos pela seguinte relação $\mathrm{BC} \geq \arccos (\mathrm{C}) /(2 \pi \sigma)$. Nesta equação, $\mathrm{C}$ é o nível de correlação utilizado na definição de banda de coerência, a qual é dada pela função $\mathrm{R}_{\mathrm{T}}(\Omega)$, que é a autocorrelação da função $T(f, t)[10]$.

Muitos autores, como Macedo [20], já tentaram relacionar banda de coerência e espalhamento de retardos em banda larga, sem que tenham conseguido um modelamento preciso.

$\mathrm{Na}$ literatura existente sobre canais UWB onde foi aplicada a equação de Gans, os valores de $\alpha$ obtidos foram bem variados. Com relação ao limiar de Fleury, constata-se uma grande quantidade de dados desrespeitando-o, em função da aquisição de valores imprecisos de banda de coerência e espalhamento de retardos por técnicas de sondagem em banda larga de resolução limitada. Com a técnica de sondagem em freqüência, em uma banda ultra larga (UWB), consegue-se reduzir a resolução melhorando a estimativa de $\alpha$. Vale destacar que ainda não se sabe a exata relação entre banda de coerência e espalhamento de retardos.

A figura 5 exemplifica o relacionamento entre o espalhamento de retardos e a banda de coerência de 0,9. Para nenhum dos locais medidos o limiar de Fleury foi ultrapassado.

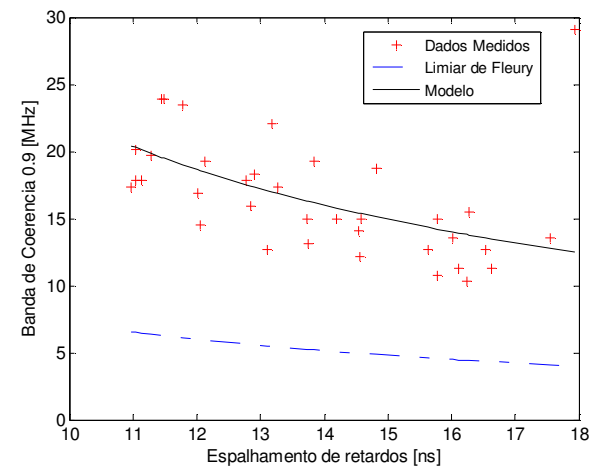

Fig. 5. Banda de coerência versus espalhamento de retardos para o local L3, em LOS, com $C=0.9$ e $\alpha=4,5$. 
A tabela 2 apresenta os resultados encontrados para: retardo médio $(\tau)$; espalhamento de retardos $\left(\sigma_{\tau}\right)$; bandas de coerência para níveis de correlação de $0,7\left(\mathrm{BC}_{0,7}\right)$ e 0,9 $\left(\mathrm{BC}_{0,9}\right)$; razão entre desvio padrão e a média do espalhamento de retardos (Razão); valor de patamar de ruído abaixo do valor de pico do sinal $(\mathrm{X})$, em $\mathrm{dB}$; limiar resultante deste patamar, em dBm; e, o número de multipercursos (NMP).

Os valores de razão encontrados deram uma boa indicativa da estacionariedade das medidas, pois não comprometeram o limiar de Fleury.

\begin{tabular}{|c|c|c|c|c|}
\hline & L1 & L2 & L3 & L4 \\
\hline $\boldsymbol{\tau}[\mathrm{ns}]$ & 28,6 & 58,9 & 52,5 & 29,7 \\
\hline $\boldsymbol{\sigma}_{\boldsymbol{\tau}}[\mathrm{ns}]$ & 12,6 & 32,0 & 13,9 & 10,9 \\
\hline $\mathbf{B C}_{\mathbf{0}, \mathbf{7}}[\mathrm{MHz}]$ & 139,2 & 66,3 & 97,3 & 83,9 \\
\hline $\mathbf{B C}_{\mathbf{0 , 9}}[\mathrm{MHz}]$ & 31,2 & 3,0 & 16,6 & 16,7 \\
\hline $\boldsymbol{\alpha}_{\mathbf{B C} \mathbf{0}, \mathbf{0}}$ & 0,6 & 0,5 & 0,8 & 1,1 \\
\hline $\boldsymbol{\alpha}_{\mathbf{B C} \mathbf{9}}$ & 2,6 & 11,2 & 4,5 & 5,6 \\
\hline Razão & 0,1 & 0,3 & 0,2 & 0,1 \\
\hline $\mathbf{X}[\mathrm{dB}]$ & 55,0 & 45,0 & 50,0 & 50,0 \\
\hline Limiar $[\mathrm{dBm}]$ & $-88,7$ & $-92,0$ & $-88,3$ & $-82,6$ \\
\hline $\mathbf{N M P}$ & 49,0 & 51,0 & 41,0 & 36,0 \\
\hline
\end{tabular}

Tabela 2. Resultados encontrados

\section{CONCLUSÕES}

A utilização da técnica de sondagem em freqüência aplicada a canais UWB mostrou-se um procedimento científico confiável e de acordo com a teoria de canais rádiomóveis, pois, para pequenos intervalos de tempo o canal pode ser considerado invariante no tempo.

A utilização da expressão proposta por Gans parece fornecer valores verossímeis para $\alpha$, devido à melhor resolução temporal proporcionada por sinais UWB. A utilização do limiar de Fleury mostrou um ajuste similar ao encontrado por Barros [22] e melhor que o obtido, em banda larga, por Macedo [21].

A janela de Blackman-Harris de 3 termos foi a que mostrou-se mais adequada para o processamento.

Os perfis de retardos de potência, que tiveram um comportamento similar nos quatro locais medidos, apresentando retardos de 400 ns e 44 multipercursos, em média. Na maioria das vezes notou-se a presença de um raio dominante, seguido de ecos com amplitudes menores.

Foi verificado que uma alteração de 1 a $5 \mathrm{~dB}$, para mais ou para menos, na definição do limiar de ruído, não altera significativamente os valores dos parâmetros de dispersão temporal do canal.

\section{REFERÊNCIAS}

[1] M. Z. Win et al. "Ultra-wide Bandwidth (UWB) Signal Propagation for Outdoor Wireless Communications". IEEE Vehicular Technology Conference, v.01, May 1997, pp. 251-255.

[2] A. Di Francesco et al. "Sounding and Modelling of the Ultra Wide-Band Channel in Outdoor Scenarios". IEEE Networking with Ultra Wide Band and Workshop on Ultra Wide Band for Sensor Networks. Networking with UWB. 2nd International Workshop, July 2005, pp. 20-24.

[3] M. Di. Renzo et al. "The Ultrawide Bandwidth Outdoor Channel: From Measurement Campaign to Statistical Modeling". 14th IST Mobile and
Wireless Communications Summit, IST Summit 2005. Dresden, Germany. 19-23 June 2005.

[4] C.W. Kim et al. "Characterization of ultra-wideband channels for outdoor office environment". IEEE Wireless Communications and Networking Conference, v.02, March 2005, pp. 950- 955.

[5] B. H. Fleury, "An uncertainly relation for WSS process and its application to WSSUS system". IEEE Transactions on Communications, v.44, n.12, December 1963, pp. 1632-1634.

[6] M. J. Gans, "A power-spectral theory of propagation in the mobile-radio environment". IEEE Transactions on Vehicular Technology, v.21, n. 01, February 1972, pp. 27-38.

[7] C. F. Souza, Análise da dispersão temporal de canais de banda ultra larga $(U W B)$ através de medidas realizadas em ambientes internos $e$ externos. Tese de Mestrado - UFF-RJ, Julho, 2006.

[8] M. G. Di-Benedetto, G. Giancola, Understanding Ultra Wide Band Radio Fundamentals, Prentice Hall Communications Engineering and Emerging Technologies Series, 2004.

[9] A. F. Molisch, "Ultra Wideband Propagation Channels - Theory, Measurements and Modeling". IEEE Transactions on Vehicular Technology, v. 54, n.5, September 2005, pp. 1528-1545.

[10] D. Parsons, The Mobile Radio Propagation Channel. 2.ed. New York: John Wiley \& Sons, 1992.

[11] H. Zaghloul, M. Fattouche, G. Morrison, "Frequency response and path loss measurements of indoor channel". Electronics Letters, v.27, n.12, June 1991, pp. 1021-1022.

[12] G.E. Carlson, Signal and Linear System Analysis. New York: John Wiley \& Sons, 1998.

[13] T. S. Rappaport, Wireless Communication Principles and Practice. 1.ed. New Jersey: Prentice-Hall, 1996.

[14] J. Keignart, N. Daniele, "Subnanosecond UWB channel sounding in frequency and temporal domain". IEEE Conference on Ultra Wideband System and Technologies, Digest of Papers, May 2002, pp. 25-30.

[15] B. P. Lathi, An Introduction to Random Signals and Communications Theory. Scranton, Pennsylvania, 1968.

[16] F. J. Harris, "On the use of windows for analysis with the Discrete Fourier Transform". Proceedings of the IEEE, v.66, n.01, January 1978, pp. 51-83.

[17] M.Z. Win, R. Scholtz, "A. Impulse Radio: How it works". IEEE Communications Letters, v.02, n.02, February 1998, pp. 36-38.

[18] D. Cassioli, M. Z. Win, A. F. Molisch, "The ultra-wideband bandwith indoor channel: from statistical model to simulations". IEEE Journal on Selected Areas in Communications, v.20, n.06, August 2002, pp. 12471257.

[19] S. M. Yano, "Investigating the Ultra Wideband Indoor Wireless Channel". IEEE Vehicular Technology Conference, v.03, May 2002, pp. $1200-1204$

[20] J. D. Taylor, Introduction to Ultra-Wideband Radar Systems. 1.ed. Boca Ranton: CRC Press, 1994

[21] L.H.G. Macedo, Sondagem em freqüência do canal indoor faixa larga. Master These - PUC-RJ, February 2002. (In Portuguese).

[22] F.J.B. Barros, R.D.Vieira, G.L. Siqueira,"Relationship between delay spread and coherence bandwidth for UWB transmission", IEEE International Conference on Microwave and Optoelectronics SBMO, July 2005 , p $415-420$. 